\title{
Shocks in Vertically Oscillated Granular Layers
}

\author{
J. Bougie, Sung Joon Moon, J. B. Swift, and Harry L. Swinney \\ Center for Nonlinear Dynamics and Department of Physics, University of Texas, Austin, TX 78712
}

(Dated: November 6, 2018)

\begin{abstract}
We study shock formation in vertically oscillated granular layers, using both molecular dynamics simulations and numerical solutions of continuum equations to Navier-Stokes order. A flat layer of grains is thrown up from an oscillating plate during each oscillation cycle and collides with the plate later in the cycle. The collisions produce layer compaction near the plate and a high temperature shock front that rapidly propagates upward through the layer. The shock is highly time-dependent, propagating through the layer in only a quarter of the cycle. We compare numerical solutions of the continuum equations to molecular dynamics simulations that assume binary, instantaneous collisions between frictionless, inelastic hard spheres. The two simulations yield results for the shock position, shape, and speed that agree well. An investigation of the effect of inelasticity shows that the shock velocity increases continuously with decreasing inelasticity; the elastic limit is not singular.
\end{abstract}

PACS numbers: $47.40 . \mathrm{Nm}, 52.35 . \mathrm{Tc}, 45.70 . \mathrm{Mg}, 62.50 .+\mathrm{p}$

\section{INTRODUCTION}

\section{A. Background}

A successful continuum description of granular media could exploit the powerful methods of standard fluid dynamics to describe a variety of granular flow phenomena, but the validity of a continuum description of granular materials has been questioned and remains an open problem [1]. Several investigators have proposed equations of motion for rapid granular flow using continuum fields number density $n$, velocity $\mathbf{u}$, and granular temperature $T\left(\frac{3}{2} T\right.$ is the average kinetic energy due to random particle motion) 2 , 3, 4]. In one approach, the interaction between grains is modeled with binary, inelastic hard sphere collision operators in kinetic theory to derive granular continuum equations to Euler [5], Navier-Stokes [ 6 , and Burnett 7] order. However, attempts to use such continuum equations to predict properties of granular media have mainly been restricted to idealized systems that are difficult to produce in experiment 2, 8, 9, 10 and to steady state or asymptotic time limits [2, 8, 9, 11]. The viability of these continuum equations for experimentally realizable, three-dimensional (3D), time-dependent granular systems has scarcely been examined. In this paper, we use 3D inelastic hard sphere molecular dynamics (MD) simulations as well as 3D simulations of continuum equations to Navier-Stokes order to investigate the strongly time-dependent properties of normal shocks in a vertically oscillated granular layer.

\section{B. Shock waves}

If the Mach number $M a$ (the ratio of the local mean fluid speed to the local speed of sound) is greater than unity at the point where a fluid encounters an obstacle, a compression wavefront is formed near the object and steepens to form a shock. In an ideal fluid with no viscosity or heat conduction, the wavefront steepens until the fields develop a mathematical discontinuity between the compressed region and the undisturbed region. In a viscous, conducting fluid, the fields vary continuously, but the front steepens until the shock thickness (the width of the region in which the fields change from the undisturbed values to the compressed values) is on the order of a mean free path.

When a supersonic fluid impinges perpendicularly onto a flat, impenetrable plate, a shock forms normal to the plate. The normal component of the mean velocity (with respect to the plate) is zero at the plate, but the velocity of the undisturbed fluid far from the plate is still supersonic, flowing towards the plate. The Mach number decreases abruptly from greater than unity to near zero as the plate is approached. The density, temperature, and pressure also change rapidly in the region of the shock front. This normal shock propagates back upstream in a direction opposite that of the upstream flow velocity.

This paper concerns normal shocks in vertically oscillating granular layers. A distinguishing feature of granular materials is that granular flows reach supersonic speeds under common laboratory conditions [2, 11, 12]. Hence shock formation, which is achieved only under extreme conditions in ordinary gases, is commonplace in granular materials. Shock propagation has been extensively studied in rarefied gases [13, 14], but the similarities and differences between shocks in ordinary fluids and in granular media is the subject of ongoing research 11, 12, 15. A recent laboratory and molecular dynamics study examined time-independent behavior of oblique shocks in granular flow between two closely spaced plates, and the results were compared with 2D simulations of Navier-Stokes order continuum equations [1].

\section{Model system}

The system consists of a layer of grains on an impenetrable plate that oscillates sinusoidally in the direction of gravity with amplitude $A$ and frequency $f$. The di- 
mensionless peak acceleration is $\Gamma=4 \pi^{2} f^{2} A / g$, where $g$ is the acceleration due to gravity. This simple oscillating system has been found to yield standing wave pattern formation 16], convection 17], clustering 18], steady-state flow fields far from the plate [19], and shocks [12]. We examine shock formation and propagation in layers that are approximately 9 particles deep as poured except in Sec. III E, where layer depth is varied. Throughout this paper, the phrase "the layer" refers to the dense region in which the volume fraction of particles is greater than $4 \%$ of the random close-packed volume fraction, $\nu_{\max }=0.65$.

When the maximum acceleration of the plate is greater than $g$, the layer leaves the plate during each cycle. When the layer is off the plate, it is cooled by inelastic collisions between particles, while the particles are simultaneously accelerated towards the plate by gravity; this leads to a large mean velocity compared to the sound speed (See Sec. III C). We show in Sec. III that when the layer contacts the plate later in the cycle, the Mach number is much greater than unity with respect to the plate, i.e., the flow is supersonic.

Shocks similar to those presented here were found for a wide range of $\Gamma$ and $f$. Here we report results obtained from a particular set of parameter values: $\Gamma=3$ and $f=$ $0.095 \sqrt{g / \sigma}$, where $\sigma$ is the diameter of a particle. This would correspond to a frequency of approximately $30 \mathrm{~Hz}$ for particles with a diameter of $0.1 \mathrm{~mm}$. The particles have a coefficient of restitution $e=0.9$, except in Sec. III $\mathrm{D}$, where $e$ is varied to investigate the effect of inelasticity on shock propagation. The number of particles in the container per unit area of the bottom plate is $10 / \sigma^{2}$, except in Sec. III E, where the number of particles is varied to investigate shocks in layers of different depths. That is, the layer would be $10 \sigma$ deep if the particles were arranged in a simple cubic lattice. In actual packings seen experimentally, 10 particles $/ \sigma^{2}$ corresponds to an average depth of approximately $H=9 \sigma$ as poured [20]. For consistency with previous investigations, we use $H$ (the depth of the layer as poured) to express the depth of the layer throughout this paper.

The values of $\Gamma$ and $f$ in this study were examined previously in experiments and MD studies of spatial pattern formation in shallow granular layers 20]. In the present simulations, pattern formation was intentionally suppressed by considering a container smaller than one full wavelength in either horizontal direction (with periodic boundary conditions at the side walls); the absence of patterns permits better visualization of the dependence of the fields on height $z$.

We present 3D continuum and MD simulations of the oscillated granular system, and analyze the behavior of the fields $n, T$, and $M a$. Section II describes the methods used to simulate and analyze the oscillatory timedependent flow. Section III reports our results, including an examination of varying inelasticity and layer depth, and Sec. IV presents our conclusions.

\section{METHODS}

\section{A. Molecular dynamics simulation}

We use an inelastic hard sphere molecular dynamics simulation that was validated in previous studies [20, 21] by comparison to experimentally observed standing wave patterns for varying control parameters. The collision model assumes instantaneous binary collisions. Linear momentum is conserved, but energy is dissipated through inelastic collisions. As in the continuum model (Sec. II B), the dissipation is assumed to be characterized by a single parameter, the normal coefficient of restitution $e$ :

$$
e=-v_{n}^{*} / v_{n}
$$

where $v_{n}=\left(\mathbf{v}_{1}-\mathbf{v}_{2}\right) \cdot\left(\mathbf{r}_{1}-\mathbf{r}_{2}\right) /\left|\mathbf{r}_{1}-\mathbf{r}_{2}\right|$, and $\mathbf{v}$ 's and $\mathbf{r}$ 's are velocity and position vectors for a pair of colliding particles before the collision (no superscript) and after the collision (with a superscript $*$ ). Particles are monodisperse, and $\left|\mathbf{r}_{1}-\mathbf{r}_{2}\right|$ and $\left|\mathbf{r}_{1}^{*}-\mathbf{r}_{2}^{*}\right|$ are identically $\sigma$. In previous studies, the normal coefficient of restitution depended on $v_{n}$ [20]. We investigate the effects of such a model in Sec. III D. However, to be consistent with the continuum model, we use a velocity-independent coefficient of restitution for most of this study. We also neglect the surface friction between particles as well as between the particles and the plate.

The particles are constrained between the bottom plate which oscillates sinusoidally between height $z=0$ and $z=2 A$, and a ceiling which is fixed at $z=200 \sigma$. In the two horizontal directions, the width is $20 \sigma$, and the boundary condition is periodic. Except in Sec. III E, 3937 particles were used; the number of particles was chosen to correspond to $H=9 \sigma$ and the total mass of the layer matches with that in the continuum simulations. In Sec. III E, 1969 particles were used to correspond to $H=4.5 \sigma$, and 5906 particles were used to correspond to $H=13.5 \sigma$. The container has the same $e$ as the particles, and the mass of the container is assumed to be infinitely large compared to that of the granular layer.

Fields are calculated by dividing the container height into small bins of size $\sigma$. The number density is the number of particles found in a bin divided by the volume of the bin. The number density $n$ from MD simulations is averaged over the same phase angle of the oscillatory state for ten cycles of the plate. The velocity $\mathbf{v}$ of each particle is used to calculate the mean velocity in each bin, $\mathbf{u}=\langle\mathbf{v}\rangle$, where the brackets represent the average over all the particles found in a bin at the same phase angle of the oscillatory state for ten cycles of the plate. Granular temperature is then defined as $T=\frac{1}{3}\left\langle|\mathbf{v}-\mathbf{u}|^{2}\right\rangle$. Throughout this paper, we use units such that the particles have mass unity. 


\section{B. Continuum simulation}

We numerically solve the continuum equations of Navier-Stokes order proposed by Jenkins and Richman 6]. These equations were derived for a dense gas composed of frictionless (smooth), inelastic hard spheres by applying Grad's 13-moment method to the inelastic Enskog-Boltzmann equation. This model yields hydrodynamic equations for number density (or equivalently, volume fraction $\left.\nu=\frac{\pi}{6} n \sigma^{3}\right)$, momentum, and granular temperature:

$$
\begin{gathered}
\frac{\partial n}{\partial t}+\nabla \cdot(n \mathbf{u})=0 \\
n\left(\frac{\partial \mathbf{u}}{\partial t}+\mathbf{u} \cdot \nabla \mathbf{u}\right)=\nabla \cdot \underline{\mathbf{P}}-n g \hat{\mathbf{z}}, \\
\frac{3}{2} n\left(\frac{\partial T}{\partial t}+\mathbf{u} \cdot \nabla T\right)=-\nabla \cdot \mathbf{q}+\underline{\mathbf{P}}: \underline{\mathbf{E}}-\gamma
\end{gathered}
$$

where the components of the symmetrized velocity gradient tensor $\underline{\mathbf{E}}$ are given by: $E_{i j}=\frac{1}{2}\left(\partial_{j} u_{i}+\partial_{i} u_{j}\right)$. The components of the stress tensor $\underline{\mathbf{P}}$ are given by the constitutive relation:

$$
P_{i j}=\left[-p+\left(\lambda-\frac{2}{3} \mu\right) E_{k k}\right] \delta_{i j}+2 \mu E_{i j},
$$

and the heat flux is calculated from Fourier's law:

$$
\mathbf{q}=-\kappa \nabla T
$$

To calculate the pressure, we use the equation of state and radial distribution function at contact proposed by Goldshtein et al. [8] to include both dense gas and inelastic effects:

$$
p=n T[1+2(1+e) G(\nu)]
$$

where

$$
G(\nu)=\nu g_{0}(\nu)
$$

and the radial distribution function at contact, $g_{0}$, is:

$$
g_{0}(\nu)=\left[1-\left(\frac{\nu}{\nu_{\max }}\right)^{\frac{4}{3} \nu_{\max }}\right]^{-1}
$$

where $\nu_{\max }=0.65$ is the $3 \mathrm{D}$ random close-packed volume fraction.

Equations (24) differ from those for a compressible, dense gas of elastic particles by the energy loss term

$$
\gamma=\frac{12}{\sqrt{\pi}}\left(1-e^{2}\right) \frac{n T^{3 / 2}}{\sigma} G(\nu)
$$

which arises from the inelasticity of collisions between particles. The bulk viscosity is given by

$$
\lambda=\frac{8}{3 \sqrt{\pi}} n \sigma T^{1 / 2} G(\nu),
$$

the shear viscosity by

$$
\mu=\frac{\sqrt{\pi}}{6} n \sigma T^{1 / 2}\left[\frac{5}{16} \frac{1}{G(\nu)}+1+\frac{4}{5}\left(1+\frac{12}{\pi}\right) G(\nu)\right],
$$

and the thermal conductivity by

$$
\kappa=\frac{15 \sqrt{\pi}}{16} n \sigma T^{1 / 2}\left[\frac{5}{24} \frac{1}{G(\nu)}+1+\frac{6}{5}\left(1+\frac{32}{9 \pi}\right) G(\nu)\right] .
$$

In marked contrast to incompressible isothermal liquid layers, $n$ and $T$ for an oscillating granular layer vary by orders of magnitude in both space and time during each cycle of oscillation; hence the viscosities and thermal conductivity are very far from constant and must be calculated at each timestep.

The equations are numerically integrated using a second order finite difference scheme on a uniform grid in $3 \mathrm{D}$ and first order adaptive time stepping. For densities near close-packed, terms of order $1 / G(\nu)$ are negligible in Eqs. (12) and (13). For reasons of numerical stability, these terms were neglected and artificial dissipation 22 was introduced in the low-density region above and below the layer. In the bulk of the layer, where the shock forms and propagates, the density near the shock is high, and the artificial dissipation has little effect on the solution.

As in the MD simulation, the granular fluid is contained between two impenetrable horizontal plates at the top and bottom of the container. We initially conducted the simulation in a cell with a ceiling that oscillates with the bottom plate at a height $200 \sigma$ above the bottom plate. For $H=9 \sigma$, where most of these simulations were conducted, an average of less than one particle per oscillation reached a height of $z \geq 80 \sigma$ in the MD simulation. Changing the height of the cell from $200 \sigma$ to $80 \sigma$ in the continuum simulation resulted in a significant speedup in computation time, but did not change the shock profile. The fractional root mean square difference over one cycle between the shock location produced in the tall cell $(200 \sigma$ high $)$ and the shorter cell $(80 \sigma$ high $)$ was less than $1 \%$. Therefore, for computational efficiency, the continuum simulations were conducted with a ceiling located at a height $80 \sigma$ above the bottom plate, oscillating with the bottom plate. In MD simulations of shallow layers, $H \lesssim 5$, many particles reach heights of $z>80 \sigma$, so we use a cell height of $200 \sigma$ in continuum simulations for $H \leq 5$ (see Sec. III E).

As in the MD simulations, we use periodic boundary conditions in the horizontal directions. Initial simulations were conducted with a container $20 \sigma$ wide in the horizontal directions, as in the MD simulation. For reasons of computational efficiency, we reduced the horizontal width of the cell to $11 \sigma$, which produced no significant 
changes in the shock behavior. The width in the horizontal directions is $11 \sigma$ for continuum simulations throughout this paper.

The continuum equations are solved in the reference frame of the container, with the container oscillations accounted for by a sinusoidal external forcing term in the momentum equation, Eq. (3). We use impenetrable boundary conditions at the plates: $u_{z}=0$ in the frame of the plate. Boundary conditions for horizontal velocities and temperature are set to match those found in the MD simulation. For most of the oscillation, their vertical derivatives at the plates were found to be negligible in the MD simulation, so for simplicity the boundary conditions used for the continuum simulation were: $\partial u_{x} / \partial z=\partial u_{y} / \partial z=0$, and $\partial T / \partial z=0$, although these derivatives are not identically zero in the MD simulation for the entire oscillation cycle of the plate.

\section{Shock tracking and shock width}

With each cycle of the plate, a normal shock wave is formed and propagates through the layer. This shock wave separates a compressed region near the plate from the undisturbed region which is still falling towards the plate. Both the undisturbed region and the compressed region vary in space and time. In Sec. III we find that when the shock is in the layer, granular temperature increases with height in the compressed region, drops quickly in the shock, and then increases again in the undisturbed region (Fig. 1). The shock is identified in simulations as the region in which the temperature falls from its maximum at the top of the compressed region to the local minimum at the bottom of the undisturbed region. The shock width $d$ is defined as the distance between the locations of this maximum and this minimum. For purposes of finding the propagation speed of the shock, the "shock location" is defined as the steepest region of temperature decrease, which is located near the center of the shock region (shaded regions in Fig. 1).

\section{RESULTS}

\section{A. Shock profile during formation and propagation}

Both simulations start with a slightly randomized flat layer near the plate which is then oscillated. A periodic state is reached after a transient state of several cycles of the plate. Although most of the particles move together as a high-density layer, some particles are always found above and below the high-density region. We define "the layer" as the region in which the volume fraction is greater than $4 \%$ of the random close-packed volume fraction $\nu_{\max }$. Below this volume fraction, there are too few particles for accurate averaging in the MD simulation.
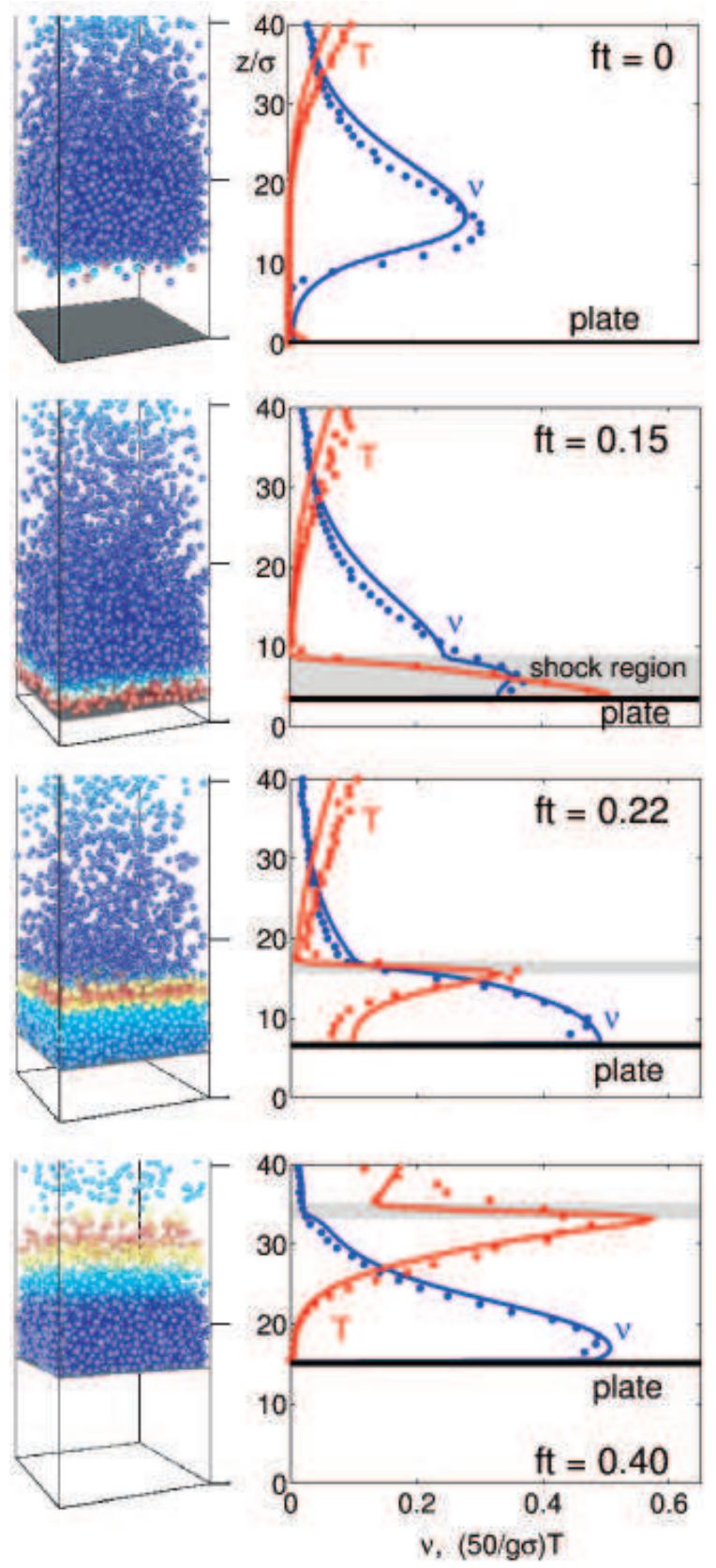

FIG. 1: Dimensionless temperature $T /(g \sigma)$ and volume fraction $\nu$ as functions of dimensionless height $z / \sigma$ at four times $f t$ in the oscillation cycle. For each time, a picture from MD simulation is shown in the left column, with individual particles color-coded according to temperature: high $T$ in red, low $T$ in blue, and the bottom plate of the container shaded solid gray. The right column shows horizontally averaged $\nu$ (blue, labeled on the bottom graph) and $T /(g \sigma)$ (red, labeled on the top graph) as functions of $z / \sigma$ (ordinate) for the same four times. The plate is shown as a horizontal black solid line, results from MD simulation are shown as dots, and continuum results are solid lines. At times when a shock is present in the graph, the shock region as located in continuum simulations (see Sec. II C) is shaded in gray. The width of the shock varies throughout the cycle, as discussed in Sec. III C. 
During each oscillation, the shock forms and propagates at the same phase angle with respect to the plate's oscillation, so the dynamics of the system are fully characterized by the time interval between time $f t=0$ and one cycle later, $f t=1$. We now investigate the formation and propagation of the shock by examining the behaviors found in MD and continuum simulations for various times $f t$ during the cycle (Fig. 1).

\section{1. $f t=0$ : The layer falls towards the plate}

At $f t=0$ the container is at its minimum height. The particles, thrown off the plate in the previous cycle, are now falling towards the plate. The temperature is nearly zero for most of the material, increasing with height only in the low density region above the layer (Fig. 1). The effects of the artificial dissipation which is used in the continuum simulation are most pronounced at this time in the cycle, while the layer is dilated and above the plate. The shock formed in the previous cycle has propagated out through the top of the layer and is not present in this picture. The difference between the MD and continuum simulation at large heights has little effect on the shock behavior at later times in the cycle.

\section{2. $f t=0.15$ : Shock forms as the layer strikes plate}

The layer now begins to strike the rising plate. The layer compresses and particle velocities are randomized by collisions, increasing $\nu$ and $T$ drastically near the plate. This results in the formation of a shock region where $\nu$ and $T$ change rapidly from the compressed, high temperature region near the plate to the dilute, low temperature undisturbed region which is still falling towards the plate. At $f t=0.15$ the shock is just beginning to form and is broad and not fully developed (see Sec. III C). During this time and throughout the formation and propagation of the shock, the shock profile from the continuum model shows remarkable agreement with the profile found in the MD simulation.

\section{3. $f t=0.22$ : Shock propagates through the layer}

The layer continues to compress on the plate, resulting in the propagation of the shock up through the layer. Here the shock is fully developed and has propagated through much of the layer, but there is still a significant number of particles falling towards the plate. The shock has steepened since $f t=0.15$, with $\nu$ and $T$ changing from the upstream to the downstream values within a distance of about $2 \sigma$. As will be discussed in Sec. III $\mathrm{C}$, the width of the shock continues to change throughout the cycle as the shock propagates through parts of the layer with different densities. A discontinuity in the derivatives of $\nu$ and $T$ appears at the leading edge of the shock, showing the boundary of the undisturbed region. Collisions cause the layer to gradually cool behind the shock, creating a lower temperature near the plate.

\section{4. $f t=0.40:$ The layer begins to leave the plate}

The shock has propagated through the bulk of the layer and now enters the very dilute region above the layer. In this region the temperature becomes uncertain in the MD simulation because there are too few particles to use in averaging. Simultaneously, the plate is approaching its maximum height, and the layer begins to leave the plate as the downward acceleration of the plate becomes larger than $g$. The layer continues to cool, setting the stage for the next oscillation.

\section{B. Shock dynamics}

The formation and propagation of the shock is shown in Fig. 2 for three values of the restitution coefficient $e$. The effect of varying inelasticity will be discussed in Sec. III D. In each case, when the bottom of the falling layer hits the plate, the center of mass of the layer is still falling towards the plate, and the layer is compressed. At this time, a shock is formed near the plate. The shock propagates up through the center of mass and out into the low density region above the layer. Then the layer leaves the plate, dilates and cools during its free flight, and once again falls towards the plate. As the shock propagates through the layer, it goes through regions of differing $\nu$ and $T$, and the shock velocity shows some slight variation with height, as seen in the curvature of the shock location (Fig. 2).

\section{Mach number and shock width}

A prerequisite for shock formation is that the local Mach number of the flow be greater than unity with respect to the object causing the disturbance. We calculate the speed of sound from MD and continuum simulations using a relation derived from the continuum equation of state Eq. (7) 23]:

$$
c=\sqrt{T \chi\left(1+\frac{2}{3} \chi+\frac{\nu}{\chi} \frac{\partial \chi}{\partial \nu}\right)},
$$

where $\chi=1+2(1+e) G(\nu)$.

As the layer falls towards the plate, it is accelerated by gravity and becomes supersonic in the reference frame of the plate. When the layer hits the plate, it is stopped by the impenetrable boundary, and the Mach number falls to nearly zero with respect to the plate (Fig. 3). The 

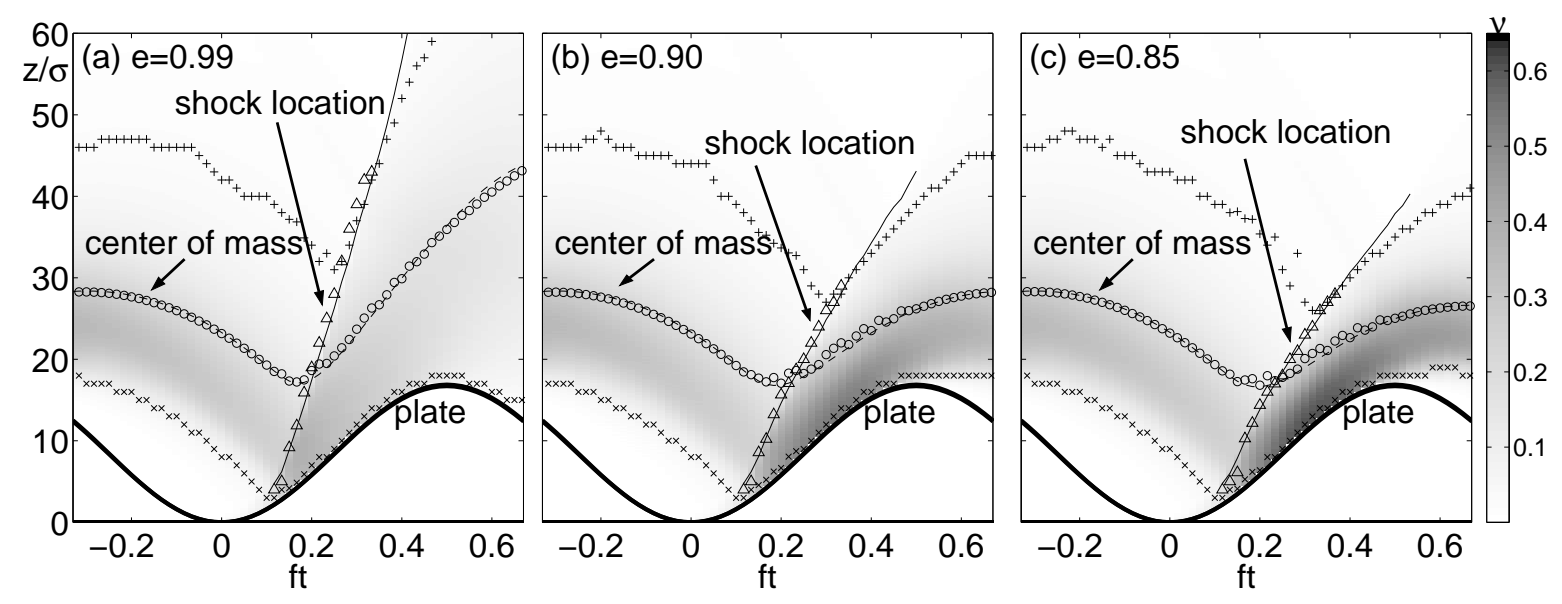

FIG. 2: Location of the shock (solid lines for continuum, triangles for MD) and the center of mass of the layer (dashed lines for continuum, circles for MD) as a function of time $f t$ during one cycle of the plate (thick solid line) for particles with (a) $e=0.99$, (b) $e=0.90$, and (c) $e=0.85$, starting from the same initial conditions at $f t=-0.33$. The plot is shaded according to the volume fraction from the continuum simulation, so that high volume fraction is dark and low volume fraction is light. The "top" and the "bottom" of the layer from MD (when the volume fraction drops to less than $4 \%$ of random close-packed) are shown as +'s and X's, respectively. The material below the shock is visibly compressed as compared to the region above the shock, as can be seen from the shading.

undisturbed region is still falling towards the plate at supersonic speeds, and the shock is formed at the boundary between the compressed (low $M a$ ) region and the undisturbed (high $M a$ ) region. The Mach number in the undisturbed region is largest near the shock where the layer has been falling the furthest under gravity, and gradually decreases with increasing height. The Mach number is directly calculated from the values of $\nu, \mathbf{u}$, and $T$, so the apparent increased discrepancy between the two simulations as compared to the discrepancy in previous figures is due to propagation of errors in these quantities in calculating $M a$.

While the shock is in the layer, the Mach number always reaches its maximum at the lower boundary of the undisturbed region. Thus the maximum value of $M a$ at a given time represents the value at the leading edge of the shock. The flows in this system are hypersonic, with $M a>10$ just above the plate immediately be-

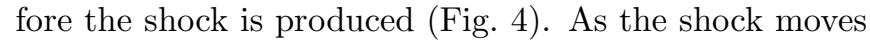
through the layer, the maximum $M a$ decreases since the fastest flow has been stopped by the shock, but the flow just above the shock remains supersonic until after the shock leaves the layer at time $f t=0.35$, and $M a$ always changes from supersonic to subsonic as the shock is crossed moving towards the plate.

Since the density of the layer varies in space and time, the mean free path of the particles in the layer changes as a function of height and time. The mean free path $\xi$ may be estimated as [24]:

$$
\xi=\frac{\sqrt{\pi / 8}}{n\left(\pi \sigma^{2}\right)}
$$

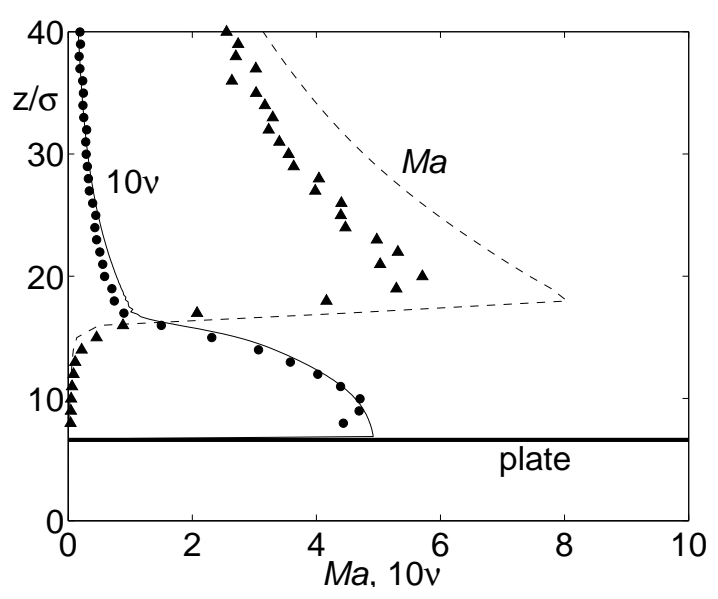

FIG. 3: Mach number $(\mathrm{Ma})$ (dashed line for continuum and triangles for MD) and rescaled volume fraction $(10 \nu)$ (solid line for continuum and circles for MD) as functions of $z / \sigma$ when $f t=0.22$. At this time the shock is fully developed and is propagating through the layer (see Fig. 1).

From $f t=0.12$ to $f t=0.17$ the shock is forming but is not fully developed. The dilute bottom of the layer hits the plate first at $f t=0.12$, so that while the initial shock is forming, a higher density region is still falling toward the plate. As higher and higher density material hits the plate, the shock width $d$ broadens (Fig. 4) as it forms from $f t=0.12$ to $f t=0.17$. After $f t=0.17$, the shock steepens until the fully formed shock is about one mean free path in width, where it remains until the shock leaves the layer at $f t=0.35$. After the shock leaves the layer, 


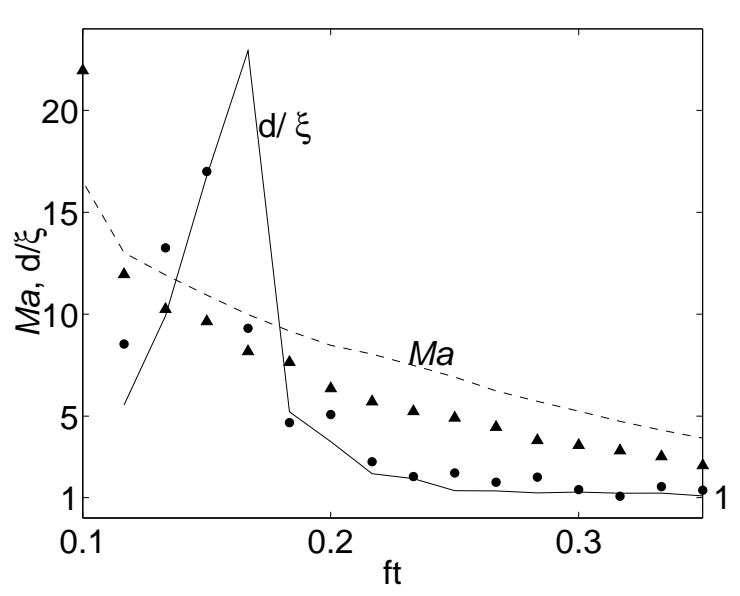

FIG. 4: The maximum Mach number $(M a)$ (dashed line for continuum and triangles for MD) and the ratio of shock width $d$ to mean free path $\xi$ (solid line for continuum and circles for $\mathrm{MD}$ ) as functions of time $\mathrm{ft}$. The shock begins forming at $f t=0.12$ and leaves the layer at $f t=0.35$.

$d$ remains approximately one mean free path, but the mean free path itself increases in the low density region above the layer, leading to a broadening of the shock front as the shock leaves the layer (compare $f t=0.40$ to $f t=0.22$ in Fig. 1).

\section{Effect of inelasticity}

Previous studies have investigated shocks in elastic and inelastic materials in the asymptotic limit of infinite time [8] and in inelastic materials at finite times [10] for a piston moving into a uniform granular gas at a constant speed in the absence of gravity. We wish to investigate the effect of inelasticity on shock dynamics at finite times in the granular shaker system. However, the oscillatory state of the layer is not appropriate for comparing a gas of elastic hard spheres to one of inelastic hard spheres. An elastic gas, which has no mechanism for removing energy, will never reach an oscillatory state since the container injects energy into the layer at each cycle of the plate. In order to investigate the effect of inelasticity on shocks in granular materials, we slightly modify the system to look at the propagation of a single shock from identical initial conditions rather than looking at oscillatory behavior.

We run simulations with different coefficients of restitution $e$, starting from identical initial conditions of a dilated layer falling towards the plate. These initial conditions are taken from the oscillatory state of the system for $e=0.9$ using the same parameters as those used in previous sections. At time $f t=-0.33$, when the layer is near the peak of its flight above the plate, the coefficient of restitution of the particles is suddenly increased or decreased.

Changing $e$ significantly changes properties of the shock and the layer following the next collision with the

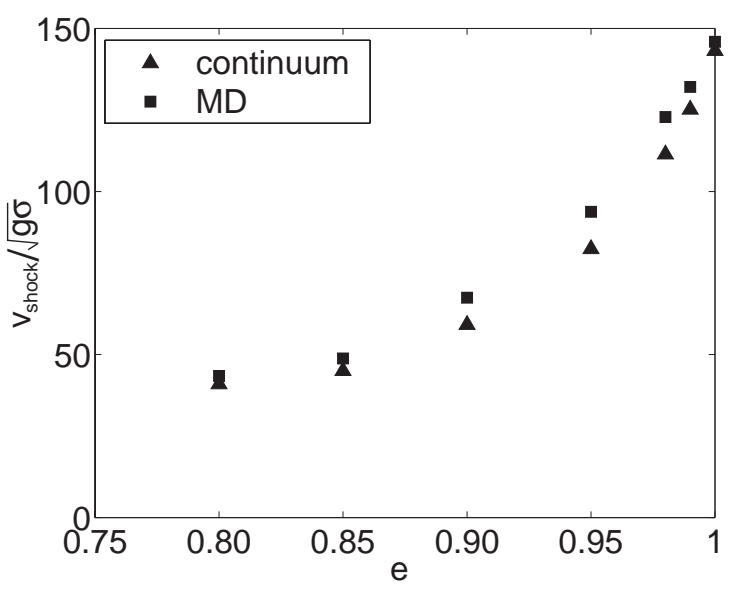

FIG. 5: Average dimensionless shock speed $v_{\text {shock }} / \sqrt{g \sigma}$ in the reference frame of the plate as a function of coefficient of restitution $e$. $v_{\text {shock }}$ is calculated as the average speed of the shock from when the shock is formed until it leaves the layer.

plate (Fig. 2). Since the initial conditions are the same for each case and the layer is in free fall, very few differences result from the change in $e$ until the layer collides with the plate. When the layer hits the plate it is compacted and many collisions occur between particles. The lower the coefficient of restitution, the more energy the particles lose through these collisions, and the more compact the layer becomes. In the case of high $e$, the layer rebounds and dilates quickly after the collision, while in the case of lower $e$, the layer remains very compact and leaves the plate almost as a solid body. The center of mass of the high $e$ layer flies very high before beginning to fall back towards the plate, while the lower $e$ value leads to a more shallow flight, with the center of mass beginning to fall earlier in the cycle. The shock trajectory exhibits curvature similar to that of the center of mass, remaining mostly straight for high $e$, but bending downward significantly for low $e$.

After the collision with the plate, we track the propagation of the shock through the layer with a range of new values of $e$. Starting from the same initial conditions, the shock starts at the same time and height regardless of $e$. However, the velocity of the shock depends on $e$, as Fig. 5 shows. Although the shock speed changes somewhat as it propagates through the layer (see Fig. 21), the average shock speed $v_{\text {shock }}$ increases monotonically with $e$ (see Fig. (5). The special case of elastic particles appears to match with the limit $e \rightarrow 1$; thus there is no qualitative difference between elastic and inelastic gases.

Throughout this paper, both MD and continuum simulations were based on a collision model in which $e$ was independent of impact velocity. A more realistic hardsphere collision model could include a coefficient of restitution $e=e\left(v_{n}\right)$ that depends on the normal component of the relative colliding velocity of the particles, $v_{n}$, such that the collisions become elastic as $v_{n}$ approaches zero. To test the effect of such a velocity- 


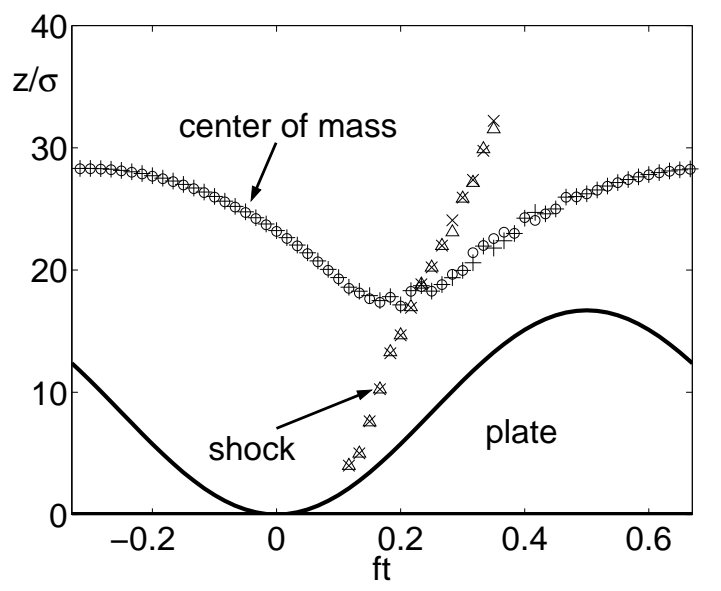

FIG. 6: Location of the shock $(\triangle$ for constant $e=0.9, \times$ for velocity-dependent $\left.e=e\left(v_{n}\right)\right)$ and the center of mass of the layer ( $\circ$ for constant $e=0.9,+$ for velocity-dependent restitution $e=e\left(v_{n}\right)$ ) as a function of time $f t$ during one cycle of the plate (thick solid line) in the MD simulation.

dependent restituition coefficient, we ran MD simulations including $e\left(v_{n}\right)$ of the same form as that used in studies of pattern formation in vertically oscillated layers [20]: $e\left(v_{n}\right)=1-0.1\left(v_{n} / \sqrt{g \sigma}\right)^{3 / 4}$ for $v_{n}<\sqrt{g \sigma}$, and $e=0.9$ otherwise. The velocity-dependence of the coefficient of restitution produces negligible changes in the shock profiles and trajectories, and less than a $1 \%$ change in the average velocity of the shock (Fig. [6). For the range of restitution values used in this paper $(0.8 \leq e \leq 1.0)$, very few particles collide with low enough relative velocity for the velocity-dependence to significantly affect the outcome. However, for velocity independent $e \lesssim 0.8$, the MD simulation was unsuccessful due to inelastic collapse, while velocity dependent $e\left(v_{n}\right)$ may be used to avoid inelastic collapse with more inelastic particles.

\section{E. Shocks in layers of different depths}

Thus far, we have restricted our investigation of shocks in oscillated layers to layer depths of $H=9 \sigma$. We now investigate how changing $H$ affects shock formation and propagation for particles with $e=0.9$.

For fluidized layers, increasing the layer depth increases the pressure compressing the layer on the plate during collision with the plate, resulting in tighter packing of the particles near the plate. For $4 \sigma \lesssim H \lesssim 15 \sigma$, increasing the layer depth increases the volume fraction of the layer near the plate, so that the trajectory of the center of mass of a layer of depth $H=13.5 \sigma$ is nearly identical to that for $H=9 \sigma$, even though the former has 1.5 times as many particles in the layer (Fig. 7(a) . Both trajectories are actually lower than the center of mass trajectory for $H=4.5 \sigma$.
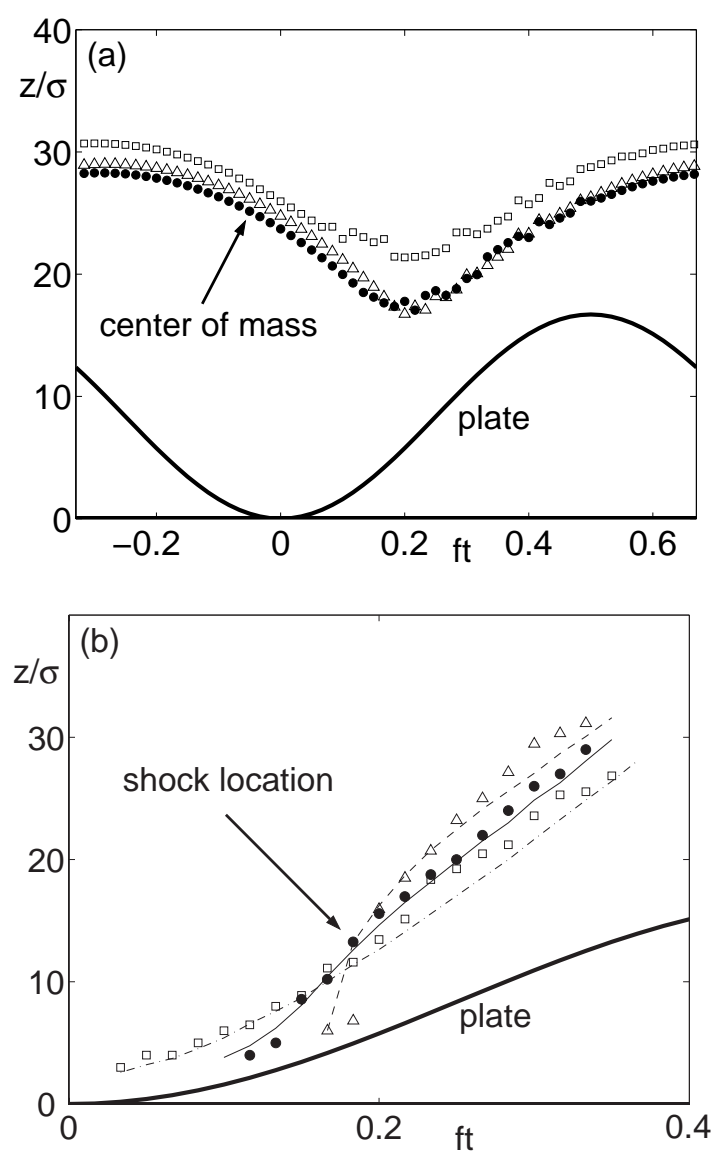

FIG. 7: (a) The height of the center of mass of the layer, and (b) the location of the shock as a function of time $f t$ for various layer depths $H$. The trajectory of the center of mass (a) is shown for a complete cycle in the oscillatory state from the MD simulation with $H=4.5 \sigma(\square), H=9 \sigma(\bullet)$, and $H=13.5 \sigma(\triangle)$. The center of mass location from continuum simulations agrees with MD to within a root mean square difference of $3 \%$ over one cycle in each of the three cases. The shock location (b) is shown for the fraction of the cycle in which the shock is in the layer, from the oscillatory state of $H=4.5 \sigma$ ( $\square$ for $\mathrm{MD},-\cdot-$ for continuum simulation), $H=9 \sigma$ (• for $\mathrm{MD},-$ for continuum $)$, and $H=13.5 \sigma$ ( $\triangle$ for $\mathrm{MD},---$ for continuum).

For $H=4.5 \sigma$, the layer is almost entirely gaseous, so much so that many particles reach heights greater than $z=80 \sigma$ in each oscillation of the plate in MD simulations with a ceiling at $z=200 \sigma$. Therefore, we use a cell of height $200 \sigma$ for continuum simulations of $H=4.5 \sigma$ (see Sec. II B).

Changing layer depth also affects the formation and propagation of shocks in the layer. For more dilute layers, the shock forms earlier in the cycle, when the lower edge of the dilute layer collides with the plate. Thus, increasing layer depth causes the shock to form later in the cycle, at which point the shock then propagates faster through the higher densities found in deeper layers (Fig. 7(b)]. 
For $H=13.5 \sigma$, the pressure on the layer during collision with the plate is so high that in some small regions near the plate, particles begin to form ordered lattices and the volume fraction increases beyond the random close-packed volume fraction. For deep layers $H \gtrsim 15 \sigma$, a significant portion of the layer is compressed into a solid lattice. The equation of state Eq. (77) does not allow volume fractions $\nu>\nu_{\max }$, so continuum simulations cannot reproduce densities greater than random closepacked found in $H \gtrsim 15 \sigma$. For $H \lesssim 4 \sigma$, the particles do not move as a coherent layer, but rather act as a gas filling the container for the entire cycle of the plate.

\section{CONCLUSIONS}

Shocks form in the vertically oscillated granular system with each collision of the layer with the plate. Properties of these shocks captured in both MD and continuum simulations show good agreement even though the system exhibits a strong time dependence and large spatial gradients. Results from the continuum simulation agree with the MD results even for ranges where the validity of the continuum equations is in question, including when $e$ is significantly less than one $(e=0.8)$ and when the volume fraction approaches close-packed. We also found that deeper layers exhibit denser packing near the plate and higher shock speeds than shallow layers. In addition, adding a simple velocity-dependence to the coefficient of restitution did not significantly change the behavior of the system. Finally, we found that increasing the coefficient of restitution causes no singular behavior in the shock velocity as $e$ approaches unity.

These results demonstrate that continuum equations can describe even a complicated three-dimensional, timedependent granular system. These results also suggest directions for further research. A more realistic model of particle collisions could include friction between particles. The simulations should be further tested by direct comparison to laboratory measurements on shocks. Finally, an investigation of the role of a shock wave in transporting energy from the plate to the layer could provide useful information about the mechanism by which oscillations of the plate provide the energy to drive flows and patterns.

\section{Acknowledgments}

We thank Daniel I. Goldman, W. D. McCormick, and Erin C. Rericha for helpful discussions. This work was supported by the Engineering Research Program of the Office of Basic Energy Sciences of the Department of Energy (Grant DE-FG03-93ER14312) and a grant from the Texas Advanced Research Program (Grant ARP 0036580055-2001).
[1] L. P. Kadanoff, Rev. Mod. Phys. 71, 435 (1999).

[2] P. K. Haff, J. Fluid Mech. 134, 401 (1983).

[3] C. S. Campbell, Annu. Rev. Fluid Mech. 22, 57 (1990).

[4] J. W. Dufty, cond-mat/0108444 (2001).

[5] A. Goldshtein and M. Shapiro, J. Fluid Mech. 282, 75 (1995).

[6] J. Jenkins and M. Richman, Arch. Rat. Mech. Anal. 87, 355 (1985).

[7] N. Sela and I. Goldhirsch, J. Fluid Mech. 361, 41 (1998).

[8] A. Goldshtein, M. Shapiro, and C. Gutfinger, J. Fluid Mech. 316, 29 (1996).

[9] J. T. Jenkins, in Physics of Dry Granular Media: Proceedings of the NATO Advanced Study Institute, edited by H.J. Herrmann, J.-P. Hovi, and S. Luding, p. 353 (1998).

[10] V. Kamenetsky, A. Goldshtein, M. Shapiro, and D. Degani, Phys. of Fluids 12, 3036 (2000).

[11] E. C. Rericha, C. Bizon, M. D. Shattuck, and H. L. Swinney, Phys. Rev. Lett. 88, 014302 (2002).

[12] A. Goldshtein, M. Shapiro, L. Moldavsky, and M. Fichman, J. Fluid Mech. 287, 349 (1995).

[13] R. Courant and K. O. Friedrichs, Supersonic Flow and Shock Waves (Interscience Publishers, Inc., New York, 1948).

[14] Y. B. Zel'dovich and Y. P. Raizer, Physics of Shock
Waves and High-Temperature Hydrodynamic Phenomena (Academic Press, New York, 1966).

[15] A. V. Potapov and C. S. Campbell, Phys. Rev. Lett. 77, 4760 (1996).

[16] F. Melo, P. Umbanhowar, and H. L. Swinney, Phys. Rev. Lett. 72, 172 (1994).

[17] J. B. Knight, E. E. Ehrichs, V. Y. Kuperman, J. K. Flint, H. M. Jaeger, and S. R. Nagel, Phys. Rev. E 54, 5726 (1996).

[18] E. Falcon, R. Wunenburger, P. Évesque, S. Fauve, C. Chabot, Y. Garrabos, and D. Beysens, Phys. Rev. Lett. 83, 440 (1999).

[19] J. J. Brey, M. J. Ruiz-Montery, and F. Moreno, Phys. Rev. E 63, 061305 (2001).

[20] C. Bizon, M. D. Shattuck, J. B. Swift, W. D. McCormick, and H. L. Swinney, Phys. Rev. Lett. 80, 57 (1998).

[21] S. J. Moon, M. D. Shattuck, C. Bizon, D. I. Goldman, J. B. Swift, and H. L. Swinney, Phys. Rev. E 65, 011301 (2002).

[22] P. J. Roache, Computational Fluid Dynamics, pp. 232237 (Hermosa Publishers, Albuquerque, 1976).

[23] S. B. Savage, J. Fluid Mech. 194, 457 (1988).

[24] K. Huang, Statistical Mechanics, 2nd Edition (John Wiley and Sons, New York, 1987). 